Produto \& Produção, vol. 6, n. 2 p. 19-30, jun. 2002

\title{
Otimização de equipes de prestação de serviços: um estudo de caso na área de suporte à micro informática
}

\author{
Luís Augusto da Costa Ilha \\ Larc Comércio e Serviços Ltda, Rua da República, 541 cj.201, Porto Alegre-RS, 90050-321, laugus1@cpovo.net \\ José Luis Duarte Ribeiro \\ Programa de Pós Graduação em Engenharia de Produção, Universidade Federal do Rio Grande do Sul, Praça \\ Argentina, 9, $2^{\circ}$ andar, Sala LOPP, Porto Alegre-RS, 90040-020, ribeiro@ufrgs.br
}

Este artigo apresenta uma abordagem que utiliza simulação para o estudo de empresas prestadoras de serviços. A abordagem é ilustrada através de um estudo de caso, realizado em uma empresa prestadora de serviços de manutenção de computadores. Utilizando a abordagem proposta, foi possível gerar cenários e simular os serviços prestados. O estudo possibilitou identificar (i) o melhor dimensionamento das equipes de front office e back office, (ii) a melhor distribuição dos funcionários, que conduz a maior produtividade, e (iii) a qualificação mais adequada para a equipe, que minimiza custos operacionais. Também foi possível realizar uma análise do lead-time, o que permitiu identificar as operações críticas e estabelecer um plano de melhorias para agilizar o atendimento ao cliente.

Palavras-chave: serviços; modelagem; simulação; produtividade.

This paper presents a simulation based approach for the study of service companies. The approach is illustrated through a case study conducted on a personal computer maintenance company. Using the proposed approach, it was possible to create scenarios and to simulate the maintenance service. The study allowed the identification of: (i) the best size for the front and back office teams, (ii) the best distribution for front/back office employees, which conduct to an increase in productivity, and (iii) the appropriate quality level for the service team, which conducts to a reduction in operational costs. It was also possible to perform an analysis of lead-times, which allows to identify the critical processes and to establish an improvement plan.

Keywords: services,modelling; simulation; productivity.

\section{Introdução}

A importância do setor de serviços tem crescido para a sociedade mundial. Albrecht e Zemke (1990) e Albrecht (1992) observam que a transição de uma economia baseada na produção para aquela baseada em serviços, é uma das tendências mais importantes do mundo empresarial moderno. O contexto econômico, observado genericamente, pode indicar que o crescimento da importância das empresas de serviços está relacionado ao impacto da era da informação e a valorização do conhecimento, assim como em outros fatores característicos deste tempo, tais como: aspectos demográficos, valorização da melhor qualidade de vida, crescente urbanização, mudanças comportamentais das pessoas e facilidades oportunizadas por avanços tecnológicos nos mais diversos setores econômicos (Gianesi, 1994).

A mudança de paradigmas no trabalho com o crescimento do setor de serviços determina, em paralelo, a valorização do conhecimento disponibilizado na mão de obra. Isso leva as empresas a reconhecerem o fato de que, na sociedade do conhecimento, a premissa mais provavelmente correta para as empresas é que elas precisam dos trabalhadores que detêm o conhecimento muito mais do que eles precisam delas (Drucker, 1999).

Um conjunto de premissas relacionadas com processos interfuncionais, profundo conhecimento da cadeia de valores, visão globalizada do negócio, conhecimen- 
to das reais necessidades dos clientes, senso de inovação, recursos humanos motivados, entre outras, necessitam de gerenciamento específico (Prahalad e Ramaswamy, 2000; Anderson, 2001; O’Connor e Veryzer, 2001).

A tecnologia disponível para o manuseio da informação está facilitando o gerenciamento dos negócios (Lev, 2000). Mas em paralelo também cresce, talvez até em proporções maiores, a necessidade de bases operacionais organizadas, disponibilizando continuamente recursos - humanos e estruturais - em condições de fornecer os serviços prometidos, preferencialmente superando a qualidade esperada (Brown e Vashistha, 2002; Berry, 2000).

Vivemos uma época em que a eficiência está cedendo lugar à eficácia ${ }^{1}$, e a concorrência crescente tem dificultado a existência das organizações em todas as áreas, em especial no setor de serviços. De acordo com Schwartz (2000), é difícil a sobrevivência daquelas empresas que ainda atuam na base do ensaio e erro. O cenário atual exige um profundo conhecimento de fatores como demanda por serviços, recursos disponíveis, capacidade produtiva, custo e qualidade do atendimento (Ghodsypour e O’Brien, 2001; Schneider e Bowen, 2000).

A melhor composição nos relacionamentos dessas componentes possibilita não só a otimização dos resultados produtivos como também a redução de perdas impostas aos clientes e as absorvidas pela empresa, validando a importância do presente trabalho, o qual apresenta uma abordagem para a otimização do uso dos recursos disponíveis. Essa abordagem será apresentada através de um estudo de caso, realizado junto à empresa Scherer Informática Ltda. Trata-se de uma empresa de pequeno porte da área de micro informática, constituída por matriz e 4 filiais distribuídas na capital e interior. Com carteira de clientes diversificada atende pessoas físicas e mercado corporativo, junto a pequenas e médias empresas. $\mathrm{O}$ negócio da empresa é atuar em projeto, instalação e suporte de manutenção com fornecimento de peças de reposição, para redes de equipamentos de informática e comunicações (hardware e softwares).

O presente trabalho considera o ponto de vista do fornecedor dos serviços e objetiva otimizar os serviços de manutenção de computadores pessoais prestados pela empresa em estudo. Nesse sentido, será necessário (i) modelar o serviço de manutenção de computadores, que envolve serviços prestados no front office (balcão de atendimento) e no back office (oficinas/laboratórios) da empresa, (ii) dimensionar a equipe de atendimento e estimar os tempos médios de conserto a partir da demanda prevista, e (iii) otimizar o serviço de manutenção de microcomputadores, através do uso de técnicas de simulação, considerando aspectos associados à produtividade e custo do fornecimento do serviço.

\section{O contexto de mercado}

O nível de exigências dos compradores tem crescido, devido ao aumento da importância que os equipamentos passaram a ter na sociedade atual. Os escritórios, empresas, profissionais autônomos e particulares, passaram a depender dos computadores em maior ou menor grau. Observa-se que, quanto maior o grau de dependência em relação aos equipamentos, maior tende a ser o nível de exigência do cliente e a insegurança quanto à qualidade e garantia dos serviços oferecidos no mercado. Também a rapidez com que novas tecnologias são disponibilizadas determina: (i) a necessidade de constante atualização, (ii) a rápida obsolescência dos equipamentos e programas, (iii) a necessidade de manutenção, e, (iv) a necessidade de auxílio e orientação na utilização de softwares e hardware tornando o mercado excepcionalmente dinâmico.

Considerando que a tecnologia é o serviço em si, o domínio que o setor possui sobre ela é significativo, dentro do que é disponível atualmente. Por outro lado, o domínio sobre as ferramentas de gestão é pequeno, com pouco conhecimento formal das técnicas de gerenciamento. O empirismo predomina. Muitas empresas possuem técnicos de nível médio e superior como gestores, que, embora bem intencionados, não dominam as técnicas administrativas.

A disponibilidade de recursos humanos constitui oportunidade à área, pois, havendo excesso de mão de obra, é possível selecionar aproveitando os melhores profissionais. Podendo constituir também ameaça, quando após desenvolver treinamento, a empresa perde o profissional para a concorrência.

O capital é indispensável para o desenvolvimento do negócio e também para o desenvolvimento e acesso a tecnologias, sendo elemento importante para a competitividade. O custo do capital é alto no mercado financeiro, comparado à rentabilidade e a própria estabilidade do setor. O repasse do custo financeiro pode inviabilizar as operações, devido às baixas margens de lucratividade e a crescente competição.

As margens de lucro são baixas devido à concorrência acirrada. Apesar disso, existem novos entrantes, pois

\footnotetext{
${ }^{1}$ Segundo Aurélio (2001) eficiência é a ação, força, virtude de produzir um efeito , enquanto que eficácia é a qualidade de quem age com eficiência e produz efeito desejado.
} 
o desemprego, fruto da não absorção da mão de obra disponível, leva as pessoas a buscar a sobrevivência no mercado informal. Nele encontram lucros, algumas vezes associados à sonegação dos impostos e ao contrabando de insumos e produtos.

As áreas concorrentes apresentam tendência a investimentos em inovações tecnológicas, como decorrência do próprio crescimento do mercado. Devido ao aumento da concorrência, o setor apresenta crescente preocupação com o gerenciamento de custos. A melhoria da qualidade e a agilidade de resposta (obtida através de redução do lead-time) são elementos importantes para a satisfação do cliente. $\mathrm{Na}$ realidade, qualidade e agilidade constituem fatores determinantes da competitividade, considerando o aumento da concorrência.

A concorrência crescente em todos os níveis, e o tempo de existência das empresas, que pode ser curto quando mal gerenciadas, formam o contexto em que é oportuno o presente estudo.

\section{Estudo do caso: desenvolvimento do modelo}

Visando retratar a realidade operacional da empresa Scherer, esta foi modelada utilizando um software de simulação, o ServiceModel. Os principais recursos deste aplicativo, que o tornam adequado ao trabalho proposto são: (i) biblioteca de construtos e possibilidades gráficas que reduzem a necessidade de programação, (ii) resultados estatísticos tabulados automaticamente, (iii) possibilidade de incluir eventos aleatórios na modelagem e, (iv) possibilidade de estabelecer regras específicas em cenários definidos para estudo. O modelo foi construído a partir dos dados informados pela empresa, contemplando as principais características das atividades desenvolvidas. Os dados que a empresa forneceu representam informações reunidas ao longo de um ano em seu software de gestão empresarial.

\subsection{Sistema a ser modelado}

Este estudo considera uma empresa de assistência técnica com características operacionais e estruturais comuns às organizações que prestam serviços de manutenção em máquinas e equipamentos.

O setor de operações da empresa é responsável pela base de produção dos serviços nos atendimentos aos clientes da empresa. A estrutura de pessoal é composta por: gerente, telefonista help desk (fornece orientações iniciais), técnicos de balcão, técnico de campo, técnicos residentes e estagiários.

\subsection{Atividade a serem analisadas}

O conjunto de atividades considerado neste estudo acontece na empresa. São os atendimentos no balcão (front office), onde o cliente assiste o serviço, e no laboratório da empresa (back office), sem a presença do cliente. É verificada a capacidade de atendimento do sistema, observando a produção obtida em diversos cenários gerados, quando alterado o número de atendentes nas posições front e back office, As atividades no front e back office são priorizadas no presente estudo, pois são elas que agregam valor e, simultaneamente, apresentam os maiores custos.

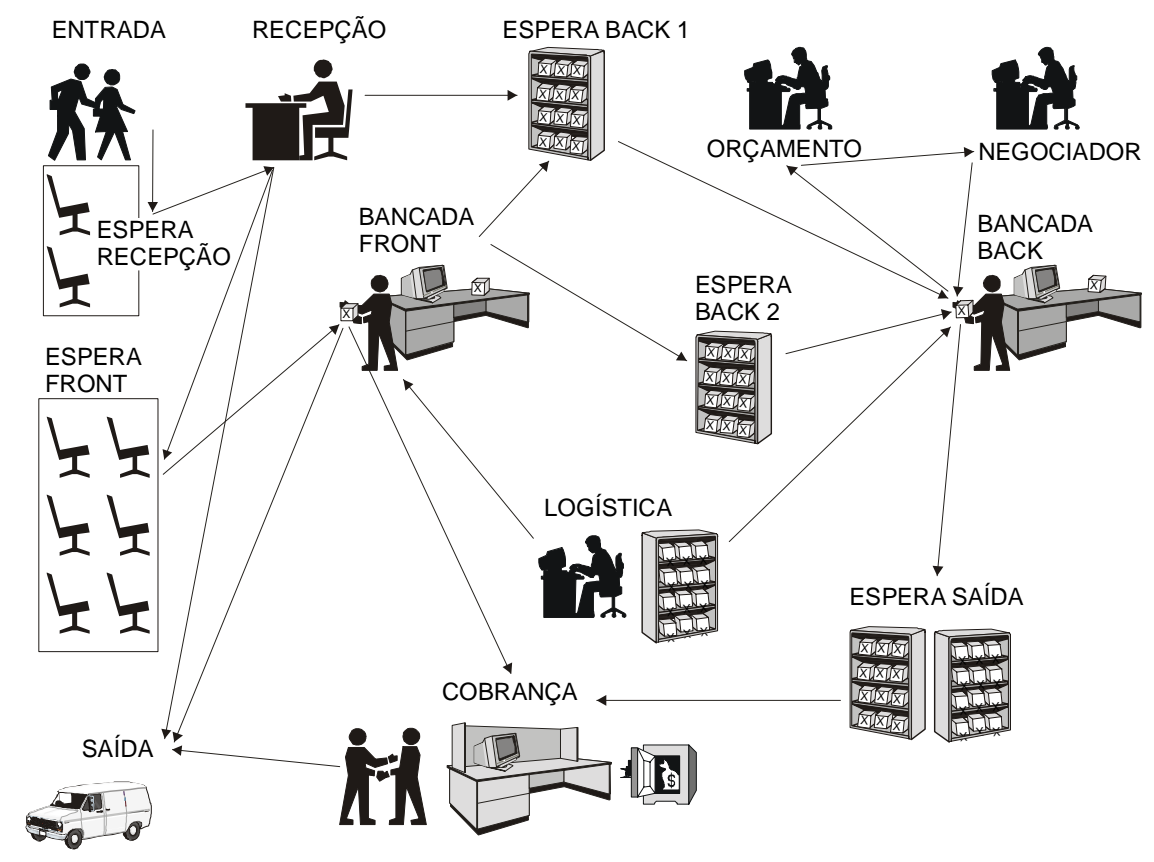

Figura 1 - Atividades na empresa Scherer Informática 


\subsection{O modelo e elementos construtivos}

O modelo está apresentado na Figura 1. Ele inclui os principais elementos existentes na empresa, que compõem o layout básico da prestação de serviços, possibilitando uma plataforma fiel à realidade, adequada para a simulação dos diversos cenários.

- Entrada: posição onde ocorrem as chegadas dos clientes no sistema modelado. Um atendente auxilia os clientes com a movimentação dos equipamentos e orienta a aguardarem na espera da recepção para chamada de atendimento.

- Espera da recepção: neste local o cliente aguarda sua chamada para atendimento.

- Recepção: aqui o cliente é recebido e consultado sobre o tipo de problema que o traz à empresa. Primeiramente, são esclarecidos a forma de trabalho e os tipos de atendimento que a organização disponibiliza. Então, o cliente escolhe o que pretende fazer: (i) desistir do conserto e sair, (ii) deixar o equipamento para orçamento ou (iii) aguardar que um técnico o atenda na hora, efetuando o conserto na sua presença.

- Espera front: tendo optado pelo atendimento imediato o cliente aguardará, neste local sua chamada para atendimento.

- Bancada front: este é o local onde um técnico atende o cliente, verificando o problema da máquina defeituosa e tentando completar o conserto. No entanto, em função do problema descrito, o cliente após as informações recebidas poderá: (i) desistir do conserto e sair ou (ii) deixar o equipamento para um orçamento mais detalhado ou ainda, (iii) deixar o equipamento aguardando peças de reposição.

- Espera Back1: neste local são armazenados os equipamentos que aguardam diagnóstico para conserto no back office.

- Espera Back2: nesta espera são armazenados os equipamentos que aguardam aprovação de orçamento e/ou aqueles que aguardam peças, havendo o serviço ocorrido no front ou no back.

- Logística: é o setor responsável pelo fornecimento das peças e componentes necessários à execução dos serviços, não importando o local (front ou back). A forma e freqüência com que acontecem os fornecimentos estão calibradas no modelo através de distribuição dos tempos na posição Espera Bk2.

- Orçamentos: local onde são montados os orçamentos, a partir das relações de componentes e serviços necessários, fornecidos pelo técnico do back office.
- Negociador: esta posição é responsável por contatar o cliente, explicar os serviços necessários, informando as custas e pedindo autorização.

- Bancada Back: posição no back office onde um técnico atua realizando inicialmente diagnósticos para consertos. Na seqüência coloca o equipamento em um armário na Espera Bk 2 e encaminha o documento contendo as informações necessárias ao orçamentista. Quando recebe um orçamento aprovado apanha o equipamento no local de depósito e inicia o conserto, requisitando peças se necessário. Uma vez pronto, o equipamento é enviado para a Espera na saída.

- Espera saída: neste local são armazenados os equipamentos prontos e testados que aguardam o cliente vir retirar ou serem remetidos.

- Cobrança: local onde os acertos financeiros com os clientes acontecem.

- Saída: posição final no processo de atendimento considera a retirada ou remessa do equipamento por conta do cliente.

- Recepcionista: recurso humano que realiza a recepção do cliente no sistema e seu acompanhamento no front, entre uma espera e outra ou na saída.

- Técnico Ft: representa o recurso humano que presta atendimento ao cliente procurando efetuar o conserto de imediato no front office. O número de técnicos Ft será alterado, para avaliar os efeitos na prestação do serviço (tempos médios e produtividade).

- Técnico Bk: recurso humano que atua no laboratório da empresa (back office). O número de técnicos Bk será alterado, para avaliar os efeitos na prestação do serviço (tempos médios e produtividade).

Na simulação do modelo deste estudo considerou-se apenas um turno de trabalho por ser a realidade de trabalho da empresa estudada. No entanto, outros dois turnos poderiam ser utilizados, talvez com vantagens na otimização do sistema.

\subsection{Os cenários}

Cenários são aquelas situações geradas por alterações feitas, em um ou mais parâmetros e/ou elementos do modelo em estudo. Dentro do objetivo de conhecer as características de comportamento do sistema modelado neste estudo, visando estabelecer um paralelo entre a capacidade de produção ideal e a praticada, o modelo criado no Service Model foi ajustado para simular diferentes cenários. 
Em uma primeira etapa os cenários foram programados para possibilitar o conhecimento do comportamento ótimo do sistema relativo ao contingente de pessoas. Em uma etapa seguinte o estudo enfatizou o conhecimento do comportamento ótimo da distribuição de determinado contingente de pessoas nas posições front e back office.

\subsection{As variáveis}

No modelo criado para este estudo, em diferentes fases, foram deliberadamente alteradas a quantidade, distribuição e qualificação do pessoal que trabalha nas bancadas do front e do back office. Vale esclarecer que o estudo concentrou-se nos funcionários do front e back office, porque tanto os custos operacionais como a capacidade produtiva da empresa dependem essencialmente desses funcionários.

Todos os dados utilizados para compor o modelo foram fornecidos pela empresa Scherer. No entanto, os dados apresentados neste artigo foram deliberadamente alterados (multiplicados por uma constante), para manter em sigilo informações consideradas estratégicas.

\subsection{Dimensionamento do contingente de técnicos nas bancadas}

Conhecendo o comportamento básico do sistema, os próximos passos envolvem a definição do número de entradas (clientes/dia) e a identificação da melhor distribuição de pessoal entre as bancadas do front e back office, ou seja, a distribuição que conduz ao melhor desempenho do sistema.

A quantidade de entradas estabelecida foi 273 máquinas por dia, o equivalente a aproximadamente 6250 máquinas por mês. Esse valor é um múltiplo da realidade atual da empresa. Inicialmente, nos primei- ros estudos de simulação, a distribuição de técnicos no front e no back foi definida em 1,0:1,2, o que corresponde à proporção usada na realidade atual da empresa.

Foram rodadas simulações para diversos cenários, investigando combinações de até cem pessoas distribuídas nas posições front e back conforme a proporção atualmente empregada, procurando identificar os pontos onde o número de clientes em filas no início do sistema inexistisse. A Figura 2 apresenta os resultados observados, indicando que uma equipe de 88 técnicos (40 no front, 48 no back) conduz a um bom desempenho no sistema, considerando como indicador básico o término de filas no início do sistema.

Outro indicador anotado que reforça essa tendência é o MTTR (Média dos Tempos Técnicos de Resolução). Foi observado que os tempos baixam ao nível de 18.000 minutos no contingente de 88 técnicos. A partir daí o aumento do contingente não apresenta efeito significativo na redução do MTTR.

\subsection{Distribuição do contingente de pessoas no front e back}

Uma vez identificado o contingente de 88 pessoas como adequado para realizar o trabalho mensal, os novos cenários passam a investigar a melhor distribuição deste contingente. Ou seja, o número de pessoas que deveriam ser alocadas às bancadas do front e back office. Foram simulados cenários onde o número de atendentes no front variou de 38 a 62, mantendose o contingente total em 88 operadores (o que equivale a dizer que o número de operadores no back variou de 50 a 26). Essa amplitude de investigação foi definida a partir de conversas com os gerentes da empresa em estudo, que informaram a faixa onde provavelmente os melhores resultados seriam obtidos.

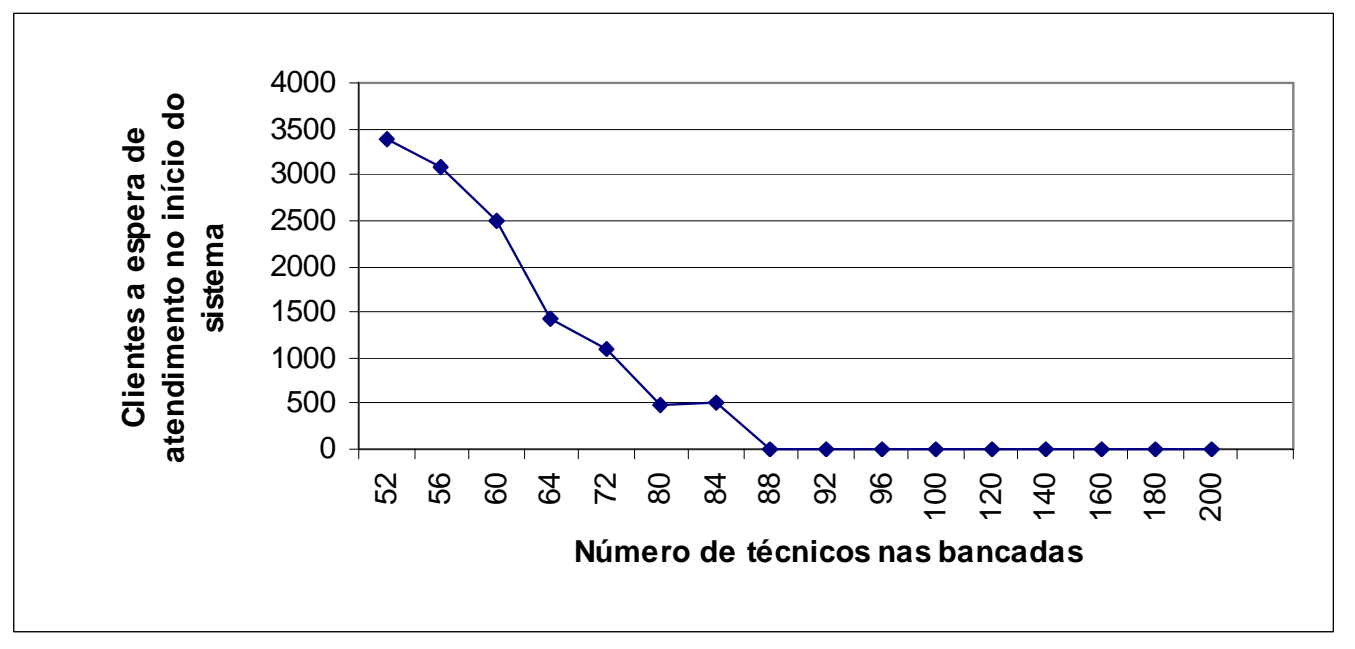

Figura 2 - Tamanho de fila na recepção em função do número total de técnicos 
Simulados os cenários nas combinações propostas, os valores observados e mostrados na Figura 3, evidenciaram que os melhores resultados estão na faixa de combinações compreendidas entre 44-44 a 53-35. O ponto ótimo do sistema está na combinação 49-39, conforme apontam as linhas de tendências das curvas geradas para diversos indicadores que serão apresentados a seguir.

\subsection{Clientes atendidos ao final do mês}

A Figura 3 revela que, as combinações do contingente de técnicos na faixa 44-44 a 53-35 apresentam a maior quantidade de atendimentos completados. A melhor combinação é o ponto 49-39, onde mesmo em dias atípicos (concentração de demanda no back ou no front) o desempenho seria bom.

\subsection{Clientes em filas na fase inicial do sistema}

Conforme visto anteriormente, a ocorrência de filas no início do sistema reduz com o aumento de contingente de técnicos no front, deixando de ocorrer quando o contingente aproxima-se de 88 técnicos. No entanto, se a distribuição dos técnicos não for adequada, o desempenho do sistema piora e novamente irá crescer a fila na entrada do sistema. Para um contingente de 88 técnicos, o desempenho fica prejudicado quando o número de técnicos no front é menor do que 44 ou quando o número de técnicos no back é menor do que 35.

\subsection{Clientes no sistema ao final do período considerado}

Os resultados das simulações realizadas demonstraram que ao final dos períodos restam clientes sem serem atendidos (residual). Esse indicador pode ser visto na Figura 4. Novamente, o desempenho do sistema piora quando o número de atendentes no front é menor que 44 ou quando o número de atendentes no back é menor do que 35.

\subsection{Percentagem de utilização das banca- das de front e back}

Os percentuais médios de utilização verificados nas bancadas de front e back podem ser vistos na Figura 5. Naturalmente, o aumento do número de técnicos no front (ou no back) conduz a ociosidade na respectiva posição. De acordo com a linha de tendência, esse indicador está levemente deslocado em relação aos demais, indicando um ponto ótimo para a combinação 44-44.

\subsection{MTTR - Média dos tempos técnicos de resolução}

A análise da Média dos Tempos Técnicos de Resolução (MTTR) em minutos novamente revela a distribuição 49-39 como aquela que apresenta o melhor desempenho (ver Figura 6).

\subsection{Percentual na relação entradas por saídas: aproveitamento}

O percentual de aproveitamento do sistema determinado pela relação saídas/entradas ao final do período simulado, também aponta um bom desempenho do sistema na faixa das distribuições 44-44 a 53-35 (ver Figura 7). O ponto de melhor aproveitamento do sistema está na distribuição 49-39, que proporciona uma margem de segurança, ou seja, uma folga para os períodos atípicos, quando o trabalho pode se concentrar mais nas bancadas do front ou do back.

Um último aspecto que merece ser destacado em relação a melhor distribuição dos técnicos nas bancadas do front e back refere-se à produtividade. Os estudos de simulação revelaram que, enquanto a distribuição atualmente utilizada na empresa (40-48) é capaz de atender 273 clientes/dia, a distribuição ótima (49-39) pode atender aproximadamente 300 clientes/ dia sem que ocorra o crescimento contínuo das filas de espera. Isso representa um aumento potencial de produtividade de aproximadamente $10 \%$.

\subsection{Influência da qualificação do contin- gente no desempenho do modelo}

Para maior conhecimento da influência dos técnicos sobre o desempenho do sistema, outros cenários foram avaliados. Os técnicos foram classificados, conforme o nível de conhecimento, em três grupos. O grupo atual, que está representado no sistema e que traduz a realidade da empresa, possui uma qualificação considerada mediana. Os estudos seguiram simulando dois novos cenários: técnicos mais qualificados e técnicos menos qualificados. Após a simulação dos novos cenários foi possível comparar os resultados, considerando tanto o desempenho do sistema quanto os custos operacionais envolvidos.

Para diferenciação da qualidade no contingente, as distribuições foram consideradas como constituídas por proporções de técnicos e estagiários. Considerando que o uso de estagiários reduz o nível técnico da equipe e os tempos de resolução, embora minimize os custos com pessoal. Essas novas simulações foram feitas utilizando a proporção ótima, ou seja, 56\% dos técnicos posicionados no front e 44\% no back. 


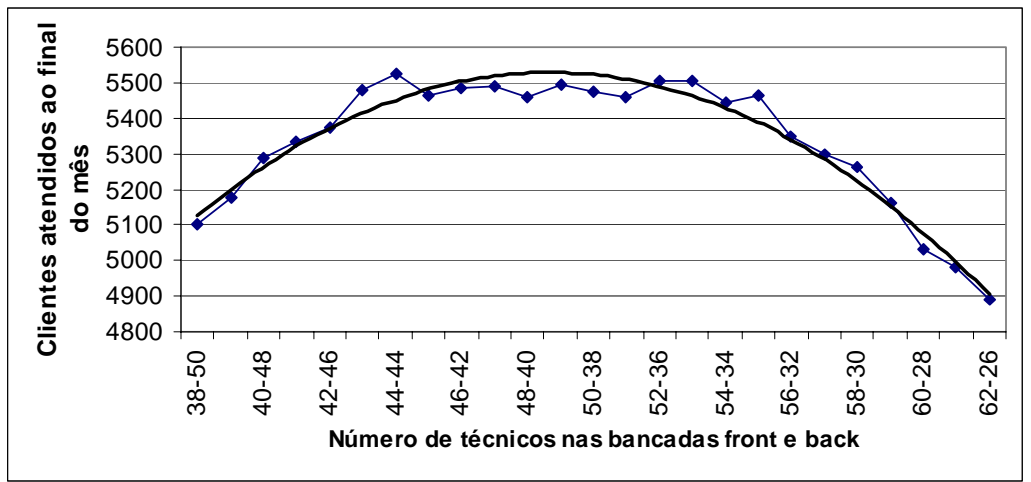

Figura 3 - Número de clientes atendidos (serviços completos) no final do mês em função da distribuição dos técnicos nas bancadas front e back

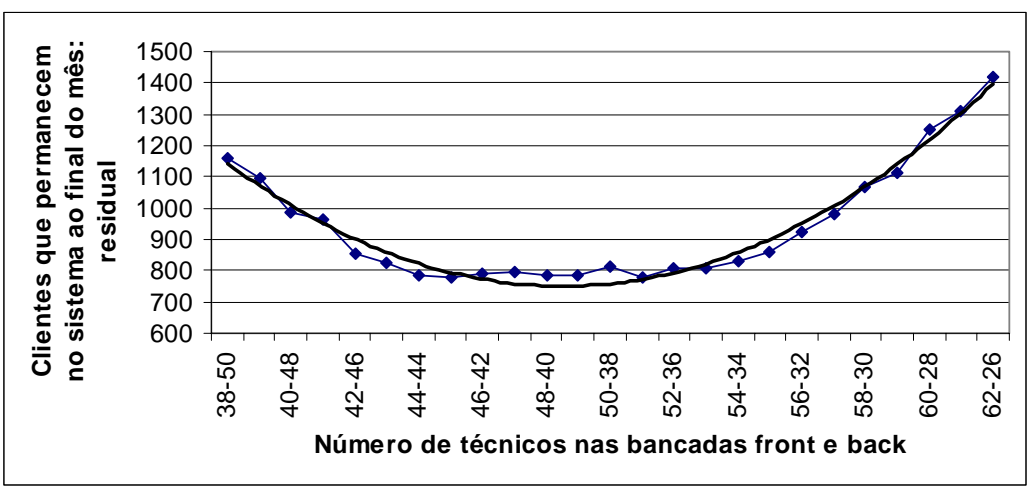

Figura 4 - Quantidade de clientes que restam sem serem atendidos em função da distribuição dos técnicos no front e back

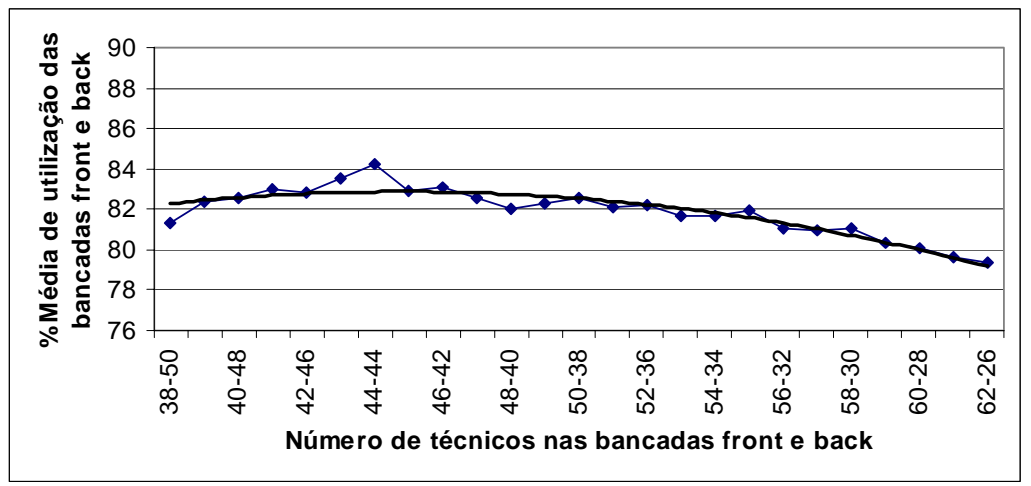

Figura 5 - Nível de utilização dos técnicos (front e back) em função da distribuição

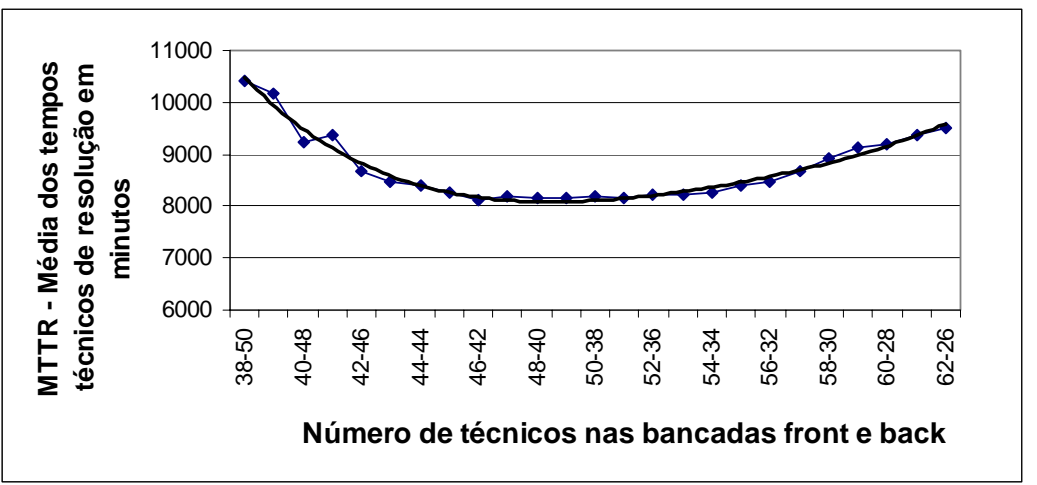

Figura 6 - MTTR em função da distribuição dos técnicos nas bancadas do front e do back 


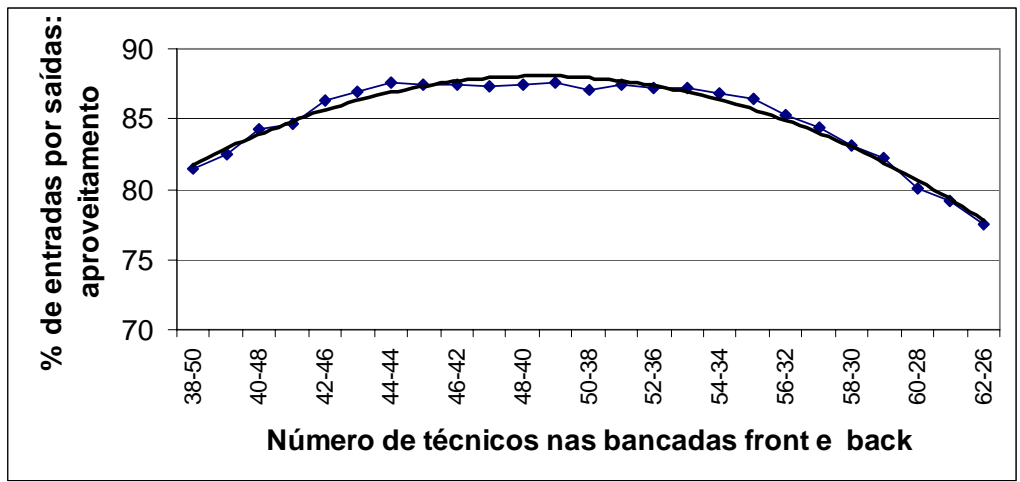

Figura 7 - Percentual de saídas/entradas, medido no final do período simulado, em função da distribuição de técnicos nas bancadas do front e back

Os grupos foram classificados em A, B e C. O grupo A caracteriza a situação considerada em todas as simulações anteriores, possui qualificação mediana, sendo composto de um técnico para cada dois estagiários. Esse grupo, conforme foi apresentado, precisa de um contingente de 88 pessoas para impedir a formação de filas, distribuídas no arranjo original. O grupo B, de menor qualificação, é composto por um técnico para cada quatro estagiários. A partir do conhecimento técnico dos gerentes da empresa, considera-se que este grupo faria o trabalho em um tempo 20\% maior. Em função disso, o número de pessoas necessárias para impedir a formação de filas (definido por simulação), seria 98 pessoas, distribuídas de acordo com a proporção ótima: 55-43. Por fim, o grupo C, apresentando maior qualificação, seria composto por um técnico para cada estagiário. A partir do conhecimento técnico dos gerentes da empresa, considera-se que este grupo faria o trabalho em um tempo 20\% menor. Em função disso, o número de pessoas necessárias para impedir a formação de filas (definido por simulação), seria 73 pessoas, distribuídas de acordo com a proporção ótima: 4132.

\subsection{Comparativo dos grupos}

Os resultados anotados nas simulações revelam que os grupos A, B e C apresentam um desempenho muito similar. Isso acontece porque as equipes menos qualificadas (mais lentas) foram compensadas por um contingente maior. Os indicadores de desempenho utilizados foram os seguintes:

- Produção total no período;

- \% de utilização das bancadas

- \% residual (clientes não atendidos no final do período)

- MTTR - Média dos Tempos Técnicos de Resolução

Assim sendo, a solução ótima é definida a partir dos custos operacionais associados a cada grupo. $\mathrm{O}$ grupo $\mathrm{B}$, menos qualificado, mobiliza um contingente maior associado a menor salário médio. O Grupo C, por outro lado, mobiliza um contingente menor associado a um maior salário médio.

Tabela 1 - Comparativo das classes A, B e C

\begin{tabular}{|l|c|c|c|}
\cline { 2 - 4 } \multicolumn{1}{c|}{} & \multicolumn{3}{c|}{ Classes } \\
\hline Indicadores & A (normal) & B (menos qualificada) & C (mais qualificado) \\
\hline Relação Técnicos/estagiários & 0,50 & 0,25 & 1,00 \\
\hline Contingente & 88 & 98 & 73 \\
\hline Melhor distribuição & $49-39$ & $55-43$ & $41-32$ \\
\hline Produção mensal & 6034 & 6040 & 6042 \\
\hline \% utilização das Bancadas & $83,59 \%$ & $88,40 \%$ & $85,71 \%$ \\
\hline \% Residual no sistema (mês) & $6,26 \%$ & $6,22 \%$ & $6,19 \%$ \\
\hline Aproveitamento & $93,74 \%$ & $93,78 \%$ & 93,81 \\
\hline MTTR & 17933 & 18940 & 18619 \\
\hline Custo operacional mensal & $280.634,49$ & $307.339,35$ & $240.524,29$ \\
\hline Custo por máquina & 46,51 & 50,88 & 39,81 \\
\hline
\end{tabular}




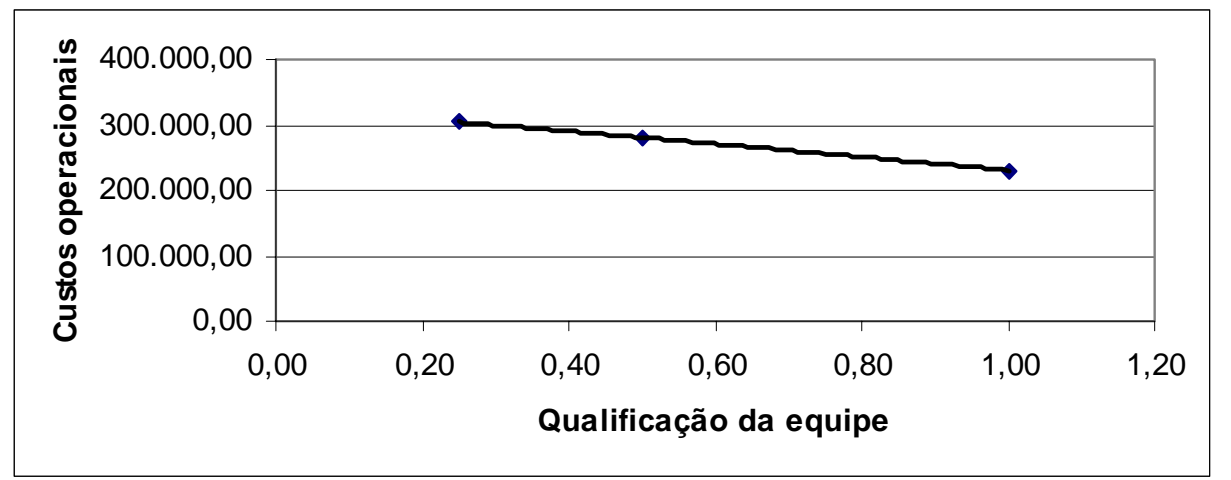

Figura 8 - Custos operacionais em função da qualificação da equipe (medida através da relação Técnicos/Estagiários)

A relação entre a qualificação do grupo e os custos operacionais aparece na Figura 8. Como pode ser visto, a partir dos dados fornecidos pelos gerentes da empresa, o Grupo mais qualificado é a melhor opção, pois apresenta o menor custo operacional total (ver Tabela 1). Como indicador de qualificação da equipe, foi usada a relação técnicos/estagiários.

Vale observar que a empresa, atualmente, trabalha com a proposta do grupo A, que, na verdade, apresenta um custo operacional superior ao Grupo C. Ao optar pela proposta do grupo C, a empresa terá a oportunidade de aumentar a sua margem de lucro ou reduzir o preço do serviço, tornando-se mais competitiva.

\subsection{Considerações relativas à sincroni- zação do sistema}

A compreensão dos custos associados ao serviço de manutenção de computadores fez com que todo o estudo fosse conduzido enfatizando as modificações no número/ distribuição/ qualificação dos técnicos. Os demais recursos como recepcionista, orçamentista, negociador, ou quem faz a cobrança foram considerados como possuindo capacidade infinita. Ou seja, na simulação, eles contam para efeito de lead time (MTTR), mas não retêm a produção. Em outras palavras, o sistema funciona como se houvesse infinitas recepcionistas, mas cada uma realizando seu trabalho em 10 minutos e retendo a máquina no processo apenas por este tempo.

Conhecendo o efeito da equipe de técnicos/estagiários sobre o desempenho do sistema, foi possível melhorar o aproveitamento. Isso foi feito alterando-se o número de técnicos, identificando o contingente necessário para evitar a formação de filas na entrada do sistema, otimizando a distribuição dos técnicos entre as bancadas do front e do back e investigando o efeito da qualificação sobre o desempenho e os custos operacionais.
Conhecido o melhor arranjo para o sistema, ou seja, Grupo C (técnicos mais qualificados), 73 pessoas, distribuídas na proporção 41-32, passa-se a um estudo do lead-time. Vale observar que, neste estudo, o lead-time (tempo de serviço) corresponde ao que vem sendo chamado de MTTR (Média dos Tempos Técnicos de Resolução), pois o serviço é um serviço de manutenção. O que se deseja é identificar as atividades que possuem maior impacto sobre o MTTR.

A Tabela 2 apresenta, em ordem decrescente, os tempos do sistema. As considerações a seguir esclarecem a análise dos tempos de atividades das posições mais significativas no sistema.

- O tempo para a aprovação do orçamento (posição negociador), na medida em que depende da autorização do cliente para liberação dos serviços, não será considerado no estudo do lead time.

- A posição da logística que retrata os tempos que os equipamentos aguardam por peças para a conclusão de consertos é preponderante na definição do lead time. Não é gargalo, pois tem capacidade de fazer inúmeros pedidos em poucos minutos, mas possui forte influência sobre o lead time (os pedidos demoram a ser atendidos) e deve ser melhor analisada.

Após a análise dos tempos das atividades, os funcionários envolvidos nos setores foram reunidos, permitindo o planejamento das seguintes ações de melhoria:

\section{Tempo aguardando cliente aprovar orçamento}

Embora o tempo aguardando o cliente aprovar o orçamento não perteça aos tempos de produção, ele é importante para a empresa porque a velocidade com que o cliente aprova os serviços influencia o fluxo de caixa da empresa. As ações propostas foram: (i) esclarecer ao cliente a relação custo-benefício do serviço proposto, visando reduzir o número de orçamentos não aprovados (atualmente, cerca de 20\% dos orçamentos realizados não é aprovado); e, (ii) criar condições de pagamento facilitadas e atraentes ao cliente. 
Tabela 2 - Tempos médios de atividade observados para as diferentes posições de trabalho

\begin{tabular}{llc}
\hline \multicolumn{1}{c}{ Posições } & \multicolumn{1}{c}{ Descrição } & Tempo (min) \\
\hline Negociador & Tempo aguardando cliente autorizar serviços & $7698^{*}$ \\
Logística & Tempo de espera por peças de reposição (Logística) & 7252 \\
Orçamentos & Tempo de preparação dos orçamentos de serviços & 2160 \\
Espera back 1 & Tempos de espera para atendimento nas bancadas back & 414 \\
Bancada back 1 & Tempos dos diagnósticos e consertos nas bancadas back & 134 \\
Bancada front 1 & Tempos dos diagnósticos e consertos nas bancadas front & 121 \\
Cobrança & Tempos das cobranças de serviços & 47 \\
Espera front & Tempo aguardando atendimento nas bancadas front & 44 \\
Recepção & Tempos de recepção e triagem inicial de clientes & 33 \\
Entrada & Chegada ao sistema & 20 \\
Espera recepção & Tempos de espera por atendimento na.recepção & 10 \\
Saída & Aguarda cliente retirar serviço & 0 \\
\hline \multicolumn{1}{c}{ Total } & & $\mathbf{1 0 2 3 5}$ \\
\hline
\end{tabular}

*Não é considerado para fins de lead-time

\section{Tempo de espera por peças}

O tempo de espera por peças exige melhorias físicas, computacionais e gerenciais:

a) Melhorias físicas: o layout da área utilizada para estocagem de peças deve ser reavaliado, com vistas a otimizar o espaço, eliminando nichos com depósitos de sucatas e espaços não aproveitados e a própria segurança no setor.

b) Melhorias de hardware e software: (i) o software de gestão deve ser completamente implantado e atualizado (interligação com filiais e novas rotinas), para a eliminação dos controles não eletrônicos, mesmo que demande adequações no sistema; (ii) o hardware utilizado pelos funcionários deve ser atualizado, objetivando agilizar as diversas operações da mídia eletrônica; (iii) as rotinas de recepção, expedição e inventários devem ser agilizadas utilizando ferramentas específicas, como por exemplo, leitora de código de barras; (iv) as rotinas e soluções operacionais do setor devem ser implementadas no FAQ (soluções dos casos freqüentes), possibilitando a auto-ajuda na execução das tarefas e facilitando o treinamento de novos funcionários.

c) Melhorias de gestão: (i) a utilização dos indicadores de desempenho nos processos do setor deve ser melhorada, a partir da utilização efetiva do software de gestão; (ii) o hábito, por parte dos funcionários, de constante uso e atualização do software de gestão deve ser incentivado.

\section{Tempo de preparação de orçamentos}

Várias das ações citadas irão auxiliar a reduzir o tempo de preparação de orçamentos. Além do que já foi citado, foram elencadas as seguintes ações:

a) Melhorias no gerenciamento eletrônico: o sistema de gestão deve possuir a lista dos preços das principais peças de reposição de cada fabricante, com atualização periódica, de forma a evitar a consulta a fornecedores a cada orçamento.

b) Melhorias de gestão: (i) o orçamentista deve ser treinado, enfatizando a utilização da mídia eletrônica; (ii) os orçamentos devem ser gerados imediatamente após a avaliação dos serviços; e (iii) devem ser criados indicadores para possibilitar o acompanhamento do trabalho do orçamentista.

\section{Comentários finais}

Esse trabalho abordou o tema simulação de serviços. $\mathrm{O}$ atendimento prestado por uma empresa da área de microinformática na manutenção de computadores pessoais, foi estudado a partir da ótica de avaliação e otimização da produtividade e dos custos dos serviços. A avaliação dos custos foi abordada considerando o ponto de vista do fornecedor dos serviços.

A abordagem proposta foi desenvolvida e apresentada a partir de um estudo de caso realizado junto à empresa Scherer Informática. Foram levantadas as informações necessárias junto à empresa para a 
modelagem dos serviços prestados no front office e no back office e para a composição das alternativas analisadas. Os cenários gerados para as simulações foram realizados com o suporte de um software específico para a modelagem de serviços: o

Servicemodel.

Esse estudo de caso possibilitou a empresa, em função dos resultados das simulações, conhecer o melhor dimensionamento das equipes de front office e back office, e também peculiaridades relativas a esse tipo de atendimento, conforme a seguir relacionadas.

- Para otimizar o desempenho do sistema, é importante dimensionar corretamente as equipes de front office e back office.

- A melhor distribuição de pessoal não pode ser feita de maneira intuitiva, pois há muita variabilidade no sistema, tornando difícil a tarefa de identificar a distribuição ótima.

- As combinações do contingente de técnicos frontback na faixa 44-44 a 53-35 apresentam os melhores desempenhos. A melhor combinação, revelada pelo estudo de simulação, é o ponto 4939, onde mesmo em dias atípicos (concentração de demanda no back ou no front) o desempenho ainda seria bom.

- A simulação revelou que a melhor distribuição dos funcionários pode gerar um aumento na produtividade de aproximadamente $10 \%$.

- Outro parâmetro que altera o desempenho do sistema é a qualificação da equipe, traduzida pelo número de técnicos e estagiários que compõem a equipe. Foram feitas simulações com equipes de diferentes qualificações, mas mantendo constante a capacidade produtiva.

- Os melhores resultados foram obtidos com a equipe mais qualificada, 73 pessoas, distribuídas na proporção 41-32, pois apresentam a mesma produtividade observada em equipes maiores e menos qualificadas e menores custos operacionais.

Além da otimização do sistema, o estudo permitiu uma análise do lead-time. Foram identificadas com precisão as atividades que tomam mais tempo, tornando o serviço mais demorado. No estudo de caso em questão essas atividades foram: (i) espera pela aprovação do orçamento, (ii) espera de peças e (iii) orçamentação. Feita a identificação dessas atividades, para cada um dos setores, foram elaboradas listas de oportunidades de melhoria, incluindo ações dirigidas a recursos humanos, software, hardware e estrutura física.
A forma que o modelo foi apresentado e estudado constitui uma proposta de abordagem para o problema em questão, ou seja, otimização de serviços considerando produtividade, custos operacionais e lead-time.

Vale ressaltar que não se pretendeu validar a abordagem proposta, uma vez que ela foi aplicada em uma única empresa. No entanto, acredita-se que ela possa ser facilmente adaptada e utilizada em muitas empresas de prestação de serviços. Isso acontece devido à generalidade das etapas que compõem a abordagem proposta, quais sejam:

a) Estudo do cenário da empresa e levantamento de todos os processos e variáveis que influenciam produtividade, custos operacionais e lead-time;

b) Modelagem e representação do sistema em um software de simulação; identificação de indicadores que qualifiquem o desempenho do sistema;

c) Análise dos cenários com suporte do software, variando os parâmetros de uma maneira estruturada, analisando as possibilidades de cruzamentos de fatores e observando a influência dos diversos fatores sobre o conjunto de indicadores;

d) Otimização do sistema a partir dos resultados dos cenários analisados considerando produtividade, custos operacionais e lead-time.

A abordagem proposta permite que as decisões sejam tomadas a partir da ótica da engenharia de produção, buscando continuamente a melhor relação custo benefício para as empresas.

\section{Referências}

ANDERSON Jr., E.G. Managing the impact of high market growth and learnig on knowledge worker productivity and service quality. European Journal of Operational Research; Amsterdam; v.134, p.508 524, 2001

ALBRECHT, K. Revolução dos serviços. Como as empresas podem revolucionar a maneira de tratar seus clientes, $3^{a}$ Edição. São Paulo: Livraria Pioneira Editora, 1992,254p.

ALBRECHT, K.; ZEMKE, R. Service America, Doing business in the new economy. New York: Warner Books, 1990.203 p.

AURÉLIO B.H.F. Novo Dicionário da Língua Portuguesa, O novo dicionário Aurélio do Século XXI, Editora Nova Fronteira, versão eletrônica, 2001. 1994. 391 p. 
BERRY, L. Em busca da boa reputação. HSM

Management, São Paulo, v.1, n.20, p.28 - 32, 2000.

BROWN, S.W.; VASHISTHA. A. Igniting the services value chain. Marketing Management, Chicago, US., v.11, p.12 - 13, 2002.

CHAMPY, J. O âmbito da ambição. HSM

Management, São Paulo, v.1, n.23, p.8 - 12, 2000.

DRUCKER, P. Desafios gerenciais para o século

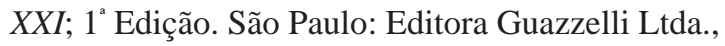
1999. 168p.

EARL, M. \& FEENY, D. O presidente ideal para a Era da Informação. HSM Management, São Paulo, v.1, n.23, p.118 - 128, 2000.

GHODSYPOUR, S.H.; O’BRIEN, C. The total cost of logistics in supplier selection, under conditions of multiple sourcing, multiple criteria and capacity constraint. International Journal of Production Economics; Amsterdam; v. 73 p. 15- 27, 2001.

GIANESI, I.G.N.; CORRÊA, H.L. Administração Estratégica de Serviços; Operações para a satisfação do Cliente. 1. Edição. São Paulo : Atlas, 1994. 233 p.

GULATI, R. \& GARINO, J. Cimentando tijolos com cliques(Get the right mix of bricks and clicks). HSM Management, São Paulo, v.1, n.23, p.90 - 100, 2000.

LEV, B. A matemática da nova economia. HSM Management, São Paulo, v.1, n.20, p.34 - 40, 2000.

O’CONNOR, G.C.; VERYZER, R.W. The nature of market visioning for technology - based radical inavation. The Journal of Product Innovation Management, New York, v.18, p. 231 - 246, 2001.

OLIVER, J.R.; THATCHER, M.E. The impact of technology investments on a firm's production efficiency, product quality, and productivity. Journal of Management Information Systems; Armonk; Fall, US, v.18, P.17 - 45, 2001.

PRAHALAD, C.K. \& RAMASWAMY, V. Como incorporar as competências do cliente. HSM Management, São Paulo, v.1, n.20, p.42 - 52, 2000.

SCHWARTZ, P. O amanhã já. HSM Management, São Paulo, v.1, n.20 p.56 - 60, 2000.

SCHNEIDER, B. \& BOWEN, D.E. O modelo das necessidades. HSM Management, São Paulo, v.1, n.23, p.64 - 72, 2000.

\section{Agradecimentos}

Os autores agradecem ao corpo gerencial da Scherer Informática Ltda, pelo suporte fornecido em todas as etapas do trabalho. Também agradecem ao CNPq pela concessão de bolsa de pesquisa. 